Please cite this article as:

Kovač-Andrić Elvira, Šorgo Glenda, Kezele Nenad, Cvitaš Tomislav and Klasinc Leo, Photochemical pollution indicators - an analysis of 12 European monitoring stations. Environmental Monitoring and Assessment, 2010, 165 (1-4): 577-583. doi: 10.1007/s10661-009-0969-7

The final publication of this article is available at link.springer.com (http://link.springer.com/article/10.1007\%2Fs10661-009-0969-7).

Published online: 19 May 2009

\title{
Photochemical pollution indicators-an analysis of 12 European monitoring stations
}

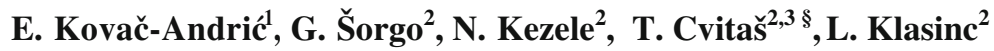 \\ ${ }^{1}$ Department of Chemistry, J. J. Strossmayer University of Osijek, Franje Kuhača 20, HR-31000 Osijek, Croatia \\ ${ }^{2}$ Ruđer Bošković Institute, HR-10 002 Zagreb, Croatia \\ ${ }^{3}$ Department of Chemistry, University of Zagreb, Horvatovac 102a, HR-10001 Zagreb, Croatia \\ e-mail: cvitas@chem.pmf.hr
}

\begin{abstract}
Indicators were devised to classify air pollution monitoring sites according to the type of expected photochemical pollution. The indicators are based on measured ozone volume fractions, the most frequently monitored component of photochemical pollution, and in particular on two contributions: one due to the ratio of daily maximum-to-minimum ozone volume fractions and the other to observed peak values. The two contributions regarded as independent are logically connected by "and" and therefore mathematically combined by multiplication. The criterion of classification is mainly described by the mentioned ratio and incidences of ozone volume fractions exceeding the limit of $80 \mathrm{ppb}$. Twelve monitoring stations within the European network (Cooperative programme for monitoring and evaluation of long-range transmission of air pollutants in Europe, EMEP) were classified according to this indicator predicting what ozone levels can be expected at the particular sites during the growth season (April through September) into three groups: clean, medium, and polluted, based on the data for the 7 years (1997 to 2003).
\end{abstract}

Keywords: Tropospheric ozone, Photochemical pollution, Air pollution indicator, Monitoring, EMEP

\section{Introduction}

There is no doubt that many urban areas and rural regions experience excessive concentrations of oxidants in the atmosphere during summer months. The main contributor to such kind of pollution is ozone formed in the atmosphere from its precursors under the influence of solar radiation. The observed severe episodes were termed photosmog. Various effects have been attributed to such kind of photochemical pollution: oxidation of sensitive materials e.g. rubber, harmful effects on plants causing decoloration and lower crop yields (Musselman and Massman 1999) and adverse effects on human health from irritations to respiratory and cardiovascular diseases (Ruidavets et al. 2005; Maitre et al. 2006; Chen et al. 2007; Sarnet and Holguin 2007) with increased numbers of hospitalizations and higher mortality (Stedman 2004; 
Fischer et al. 2004; Ko et al. 2007; West et al. 2007; Zanobetti and Schwartz 2008).

Worldwide, strong efforts are being made to reduce photochemical pollution and, since ozone is the most regularly monitored component of this kind of pollution, most air quality recommendations are based on its volume fraction (or mass concentration) in ambient air. Recently established target values and long-term objectives for the protection of human health and vegetation are frequently being exceeded at different locations, especially in urban agglomerations of many countries. Ozone fractions at various locations and regions reveal a strong spatial and temporal variability and significant differences between rural, suburban, and urban sites within the same region. There are difficulties in establishing relatively simple indicators which would enable meaningful comparisons of air quality at different locations.

In some countries expected pollutant concentrations or volume fractions in ambient air are reported together with weather forecast data, particularly when air quality standards are likely to be violated, to indicate the degree of well being when residing outside.

It is not easy to find a simple representative value to describe photochemical pollution, a complex state in ambient air involving various trace species at excessive concentrations. It is likely that not all the relevant components have been identified yet, but only a few of the known ones are monitored on a regular basis. Furthermore, the concentrations of photochemical pollution com- ponents as well as their ratios often tend to vary dramatically during $24 \mathrm{~h}$ of the day.

It is the aim of the present article to analyze different patterns of behavior of the leading species in photochemically polluted air, namely ozone, in order to find a simple way of classifying types of monitoring sites with respect to probability and extent of expected photochemical pollution. The main advantage of choosing ozone is that it is relatively frequently and reliably monitored at many stations.

\section{Stations}

Twelve stations of the European network (Cooperative Programme for Monitoring and Evaluation of Long-Range Transmission of Air Pollutants in Europe, EMEP) were selected for the analysis mainly for some comparative features with the monitoring sites in Croatia. They are listed in Table 1 together with their geographical coordinates. The geographical locations are indicated on the map in Fig. 1, but full geographical coordinates can be found on the web (www.nilu.no/projects/ccc/network.html). Among the stations there is only one at high altitude (Krvavec, 9), two are at medium altitude (Kosetice, 6 and Payerne, 10) while all the others are at low altitudes.

Mass concentrations for ozone in micrograms per cubic meter are available on the Web (www.nilu.no/projects/ccc/network.html) and we have chosen the summer periods (1 April to 30

Table 1 EMEP monitoring stations selected for the analysis

\begin{tabular}{lllrr}
\hline No. & Station, country code & Latitude & Longitude & Altitude \\
\hline 1 & Bottesford, GB & $52.56^{\circ} \mathrm{N}$ & $0.49^{\circ} \mathrm{W}$ & $32 \mathrm{~m}$ \\
2 & Vezin, BE & $50.30^{\circ} \mathrm{N}$ & $4.59^{\circ} \mathrm{E}$ & $160 \mathrm{~m}$ \\
3 & Neuglobsow, DE & $53.09^{\circ} \mathrm{N}$ & $13.02^{\circ} \mathrm{E}$ & $62 \mathrm{~m}$ \\
4 & Diabla Gora, PL & $54.09^{\circ} \mathrm{N}$ & $22.04^{\circ} \mathrm{E}$ & $157 \mathrm{~m}$ \\
5 & Jarczew, PL & $51.19^{\circ} \mathrm{N}$ & $21.59^{\circ} \mathrm{E}$ & $180 \mathrm{~m}$ \\
6 & Kosetice, CZ & $49.35^{\circ} \mathrm{N}$ & $15.05^{\circ} \mathrm{E}$ & $534 \mathrm{~m}$ \\
7 & K-Puszta, HU & $46.58^{\circ} \mathrm{N}$ & $19.35^{\circ} \mathrm{E}$ & $125 \mathrm{~m}$ \\
8 & Illmitz, AT & $47.46^{\circ} \mathrm{N}$ & $16.46^{\circ} \mathrm{E}$ & $117 \mathrm{~m}$ \\
9 & Krvavec, SI & $46.18^{\circ} \mathrm{N}$ & $14.32^{\circ} \mathrm{E}$ & $1,720 \mathrm{~m}$ \\
10 & Payerne, CH & $46.48^{\circ} \mathrm{N}$ & $6.57^{\circ} \mathrm{E}$ & $510 \mathrm{~m}$ \\
11 & Peyrusse Vielle, FR & $47.22^{\circ} \mathrm{N}$ & $0.06^{\circ} \mathrm{E}$ & $236 \mathrm{~m}$ \\
12 & Montelibretti, IT & $42.06^{\circ} \mathrm{N}$ & $12.38^{\circ} \mathrm{E}$ & $48 \mathrm{~m}$ \\
\hline
\end{tabular}


Fig. 1 Locations of the investigated European stations: 1 Bottesford, 2 Vezin, 3 Neuglobsow, 4 Diabla Gora, 5 Jarczew, 6 Kosetice, 7 K-puszta, 8 Illmitz, 9 Krvavec, 10 Payerne, 11 Peyrusse Vielle, 12 Montelibretti

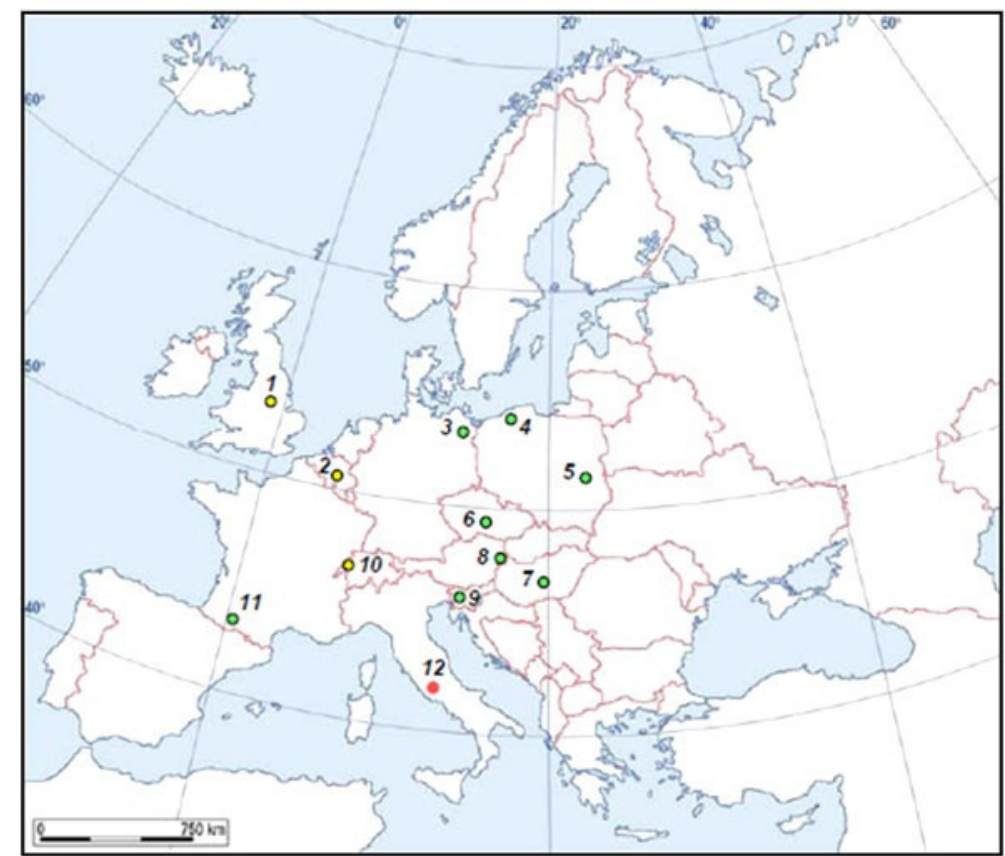

September) of 7 years 1997 to 2003 for the analysis. More recent data were not available for all stations.

\section{Method}

Relatively comprehensive information on ozone behavior at a particular site can be obtained by plotting average daily behavior (Butković et al. 1990), or even better by plotting the so called boxand-whiskers diagrams (Jeftić and Cvitaš 1991). However, such plots require additional work to produce and relatively high background knowledge to understand.

Much simpler would be giving just the average value of all the measured hourly average volume fractions. The daily, weekly, or monthly average concentration of a pollutant is a good indicator when the concentration is roughly constant during that period of time. Ozone, however, shows a characteristic diurnal behavior with maximum concentrations in the early afternoon hours and minimum values in the morning and evening hours. This variation is much more pronounced in urban than in rural sites. At high altitudes near the top of the boundary layer the variation tends to disappear altogether.

Standard deviations would provide interesting additional information provided the original data are reasonably normally distributed which is rarely satisfied. It can be expected that at high altitudes, where hardly any diurnal variations are observed, the measured values will congregate much more closely to the mean value, than at low altitude sites, giving rise to small standard deviations. Urban centers with strong emission sources of primary pollutants have a high frequency of both low and high concentrations and will therefore show relatively large standard deviations.

Typical behavior of ozone volume fractions during a photosmog episode involves very low, even zero values in absence of light owing to ozone consuming reactions with primary pollutants and very high values in the early afternoon hours. Such behavior can best be characterized by the ratio of daily maximum and minimum values. By taking the average of the maximum/minimum ratios for each day during the whole period a simple characteristic of the type of monitoring site is obtained. When minimum ozone volume fractions were found to be zero, they were replaced by $0.4 \mathrm{ppb}$ (i.e. just below half of the usual 
measurement uncertainty) in order to avoid division by zero. This criterion was applied previously in some instances (Cvitaš et al. 1995).

Air quality standards were earlier usually based on peak hourly or half-hourly values and the frequency of their incidence. We have tried to take this also into account. We determined the maximum hourly average ozone volume fraction for each day and calculated the average of such daily maxima for the whole season.

Two more parameters were chosen on the basis of ozone volume fractions in excess of a frequently chosen air quality limit: an hourly average of $80 \mathrm{ppb}$. One gives the total volume fraction in excess of the chosen limit for the whole season (in ppb·h), the other just the number of hours the limit was exceeded.

\section{Results}

The described parameters were calculated for the 12 EMEP stations and the average values based on hourly average volume fractions calculated for seven seasons are compared in Table 2.

By comparing the parameters some interesting features can be identified. As expected the average value in column 2 of Table 2 does not vary strongly. By far the highest value was found at the high-altitude station Krvavec where no low values occur. Low average values were measured in the western stations Bottesford and Vezin, while all the others lie within the range of 30 to $40 \mathrm{ppb}$.
The standard deviations (column 3) are almost constant between 11 and $17 \mathrm{ppb}$, except for the high altitude station 9 where it is significantly lower and the obviously polluted site Montelibretti (12) where it is much higher.

The maximum to minimum ratios (column 4) which were used before for characterizing monitoring sites (Cvitaš et al. 1995) classify the stations in the range between 1 and 50. The high values are representative of sites with significant local photochemical production as well as ozone consumption. Stations Montelibretti (12), Payerne (10), Vezin (2), and Bottesford (1) appear to be leading in this respect.

The incidence of high ozone volume fractions can be represented in various ways. We have chosen three: (1) the average maximum value, $M$, is obtained as the average of daily maximum values, (2) the total excess is calculated as the sum of hourly volume fractions in excess of $80 \mathrm{ppb}, E$, the so called accumulated ozone time (AOT80) (Wayne 2000), and (3) the duration of ozone volume fractions exceeding the limit of $80 \mathrm{ppb}, D$, in hours. These values are given in columns 5, 6, and 7 of Table 2. Stations Montelibretti (12), K-puszta (7), and Krvavec (9) show the highest values irrespective of the parameter chosen. Parameters (2) and (3) show hardly any difference in classifying the stations and since (3) is simpler we gave it preference.

The next step consists in the combination of two criteria: the one given by the maximum-tominimum ratio and the other representing adequately the peak values. The two contributions of
Table 2 Statistical parameters obtained from hourly average volume fractions of ozone given as averages of seven summer periods of 1997 to 2003

For stations Krvavec (9) and Peyrusse Vielle (11) only six seasons were taken into account. Data for 1998 at Krvavec and 1997 at Peyrusse Vielle were unavailable.

\begin{tabular}{lllllcc}
\hline $\begin{array}{l}\text { Station } \\
\text { no. }\end{array}$ & $\begin{array}{l}\text { Average } \\
A / \mathrm{ppb}\end{array}$ & $\begin{array}{l}\text { Standard } \\
\text { deviation/ppb }\end{array}$ & $\begin{array}{l}\text { Ratio } \\
\max / \min R\end{array}$ & $\begin{array}{l}\text { Average } \\
\max M / \mathrm{ppb}\end{array}$ & $\begin{array}{l}\text { Total } \\
\text { excess/ppb }\end{array}$ & $\begin{array}{l}\text { Duration of } \\
\text { excess } D / \mathrm{h}\end{array}$ \\
\hline 1 & 26.9 & 13.6 & 13.6 & 42.4 & 106.4 & 12.7 \\
2 & 27.7 & 17.4 & 18.4 & 48.1 & 304.3 & 32.7 \\
3 & 32.9 & 15.7 & 9.4 & 49.7 & 134.1 & 16.4 \\
4 & 34.2 & 13.9 & 7.0 & 48.4 & 0.1 & 0.1 \\
5 & 32.7 & 14.9 & 5.0 & 49.3 & 62.9 & 1.3 \\
6 & 40.1 & 13.4 & 2.9 & 53.6 & 60.6 & 9.9 \\
7 & 43.3 & 17.1 & 4.3 & 63.1 & 339.9 & 59.7 \\
8 & 40.5 & 14.8 & 3.5 & 58.0 & 214.1 & 28.6 \\
9 & 56.0 & 10.0 & 1.2 & 64.2 & 349.0 & 66.0 \\
10 & 34.8 & 17.1 & 16.2 & 55.0 & 147.0 & 23.3 \\
11 & 36.0 & 11.3 & 2.1 & 47.2 & 22.0 & 5.2 \\
12 & 32.0 & 22.9 & 45.9 & 66.9 & $1,233.4$ & 108.6 \\
\hline
\end{tabular}


independent criteria for predicting the probability of the first and second to be fulfilled have to be combined by multiplication. The ratio is always a value greater than 1 and rarely exceeding 50 . Multiplication by a factor of the order of one would be desirable to retain the same order of magnitude for the product.

Therefore we chose the average maximum value $(M)$ divided by the average volume fraction (A) - a factor in the range between 1 and 2.2. The product as an indicator characterizing a monitoring site with respect to photochemical pollution,

$P_{1}=R M / A$

varies between 1.5 and 100 for the examined 12 stations. The ranking of stations with respect to $P_{1}$ is given in Table 3 on the left-hand side. The most polluted station in a class of its own is Montelibretti (12). The stations Payerne (10), Vezin (2), and Bottesford (1) represent a medium class where photosmog episodes can occasionally occur, but probably very rarely. Three stations are clearly very clean with respect to photochemical pollution: Krvavec (9), Peyrusse Vielle (11), and Kosetice (6). The remaining EMEP sites fall between the two extremes.

The second indicator was calculated by a factor taking into account the number of hours when the air quality limit of $80 \mathrm{ppb}$ was exceeded. In order to avoid multiplication by zero when $80 \mathrm{ppb}$ was not exceeded at all, the chosen factor was $\left(1+7 D / N_{\mathrm{d}}\right)$ where $D$ is the number of hours with

Table 3 The ranking of 12 EMEP stations according to the devised indicators given as averages ( \pm standard deviation) for seven summer seasons 1997 to 2003

\begin{tabular}{lrrr} 
Station no. & Indicator $P_{1}$ & Station no. & Indicator $P_{2}$ \\
9 & $1.8 \pm 0.2$ & 11 & $2.5 \pm 1.0$ \\
11 & $2.9 \pm 0.1$ & 6 & $3.5 \pm 1.3$ \\
6 & $3.9 \pm 0.2$ & 9 & $3.8 \pm 3.0$ \\
8 & $4.7 \pm 0.7$ & 5 & $5.1 \pm 0.7$ \\
7 & $6.1 \pm 1.4$ & 8 & $5.4 \pm 3.9$ \\
5 & $7.3 \pm 1.0$ & 4 & $7.2 \pm 2.7$ \\
4 & $10.1 \pm 3.8$ & 7 & $13.4 \pm 8.4$ \\
3 & $14.1 \pm 3.5$ & 3 & $14 \pm 10$ \\
1 & $21.6 \pm 9.6$ & 1 & $17.8 \pm 7.8$ \\
10 & $26 \pm 13$ & 10 & $25 \pm 12$ \\
2 & $32.2 \pm 6.3$ & 2 & $40 \pm 33$ \\
12 & $97 \pm 43$ & 12 & $220 \pm 94$ \\
\hline
\end{tabular}

ozone volume fractions in excess of $80 \mathrm{ppb}$ and $N_{\mathrm{d}}$ is the number of monitoring days per season. The number of hours with such excessive ozone volume fractions in a season is often above 100 which represent roughly $50 \%$ of the days in a semi-annual season. This makes the contribution of high values fall into a range of 1 (when the air quality limit was never exceeded) to 8 (when it was exceeded on the average for $1 \mathrm{~h}$ per day). If the air quality limit was exceeded $1 \mathrm{~h}$ per week on the average, the formula would lead to a factor of 2 . The second indicator for classifying the sites can thus be defined as

$$
P_{2}=R\left(1+7 D / N_{\mathrm{d}}\right)
$$

This leads to a very similar ranking of stations as the previous method (Table 3, right-hand side). This method however gives a clearer grouping into three classes: clean $\left(P_{2}<10\right)$, medium $(10<$ $\left.P_{2}<40\right)$, polluted $\left(P_{2}>100\right)$. In addition it gives a higher weight to the effect related peak ozone fractions in ambient air.

The ranking of stations on the basis of the two calculated indices as described above for the 12 stations are compared in Table 3. The first method is somewhat simpler than the second and does not alter the ranking of the stations from that on the basis of the average maximum-to-minimum ratio. The second method yielding $P_{2}$ gives a higher weight to the peak values but nonetheless the results i.e. the ranking of stations remains practically unchanged. The high altitude station Krvavec (9) changes from the first position, when ranking according to $P_{1}$, to third position when ranking with respect to $P_{2}$. The ranking of the Hungarian station 7 changes drastically because of the incidence of peak values exceeding $80 \mathrm{ppb}$ is very frequent. It is the second site in this respect as seen from Table 2 .

\section{Discussion}

Air pollution indices are usually defined in order to give an indication of air quality based on currently measured values at a particular location and time and to inform the public whether the current ambient air can be regarded as good or unhealthy (McNaught and Wilkinson 1997) for dwelling 
outdoors. The present method was devised in order to classify sites according to the type of pollution that can be expected in a particular season at the given site, i.e. on the probability of photochemical pollution episodes to occur during the summer season. Twelve stations from the EMEP network were examined in this respect for their behavior during the summer or growth season.

The parameters found most useful with respect to simplicity of how they can be calculated and the information they provide were the average daily maximum-to-minimum ratio and the average daily maximum ozone volume fraction. By multiplication of these factors an indicator is obtained according to which the stations can be classified into three or four groups. One group represents relatively clean air stations with little local photochemical production and scarce episodes exceeding the air quality standard for ozone given by an hourly average of $80 \mathrm{ppb}$. These stations are characterized by an indicator value of less than 10. The second group represents stations where there is local photochemical production of ozone and the hourly average ozone volume fraction exceeds the air quality limit occasionally. The corresponding value of the indicator lies in the range of 10 to 50 . The third group represents stations with frequent photosmog episodes, a high local photochemical production and significant local primary pollutant sources. Among the investigated EMEP stations only the station Montelibretti in Italy falls into that class with an indicator greater than 100 .

Based on the present study the second method yielding $P_{2}$ provides a clearer classification of stations with respect to most frequently encountered air pollution problems in the summer period. It gives greater weight to effect-related peak ozone data i.e. to values exceeding a standard at which effects on humans can be expected.

The classifications were based on the average values for seven seasons. However, in most stations no large changes are to be expected when applying the method for individual seasons. Some exceptions should, however, be noted. These are most easily identified by the large relative deviations in the indicator $P_{2}$ as given in Table 3 . In the cases of stations Krvavec (9) in Slovenia, Illmitz (8) in Austria, Neuglobsow (3) in Germany, and
Vezin (2) in Belgium the relative standard deviations amount to more than $70 \%$. The first two stations are relatively close, separated by ca. $280 \mathrm{~km}$, and a common cause might be expected. Indeed, the $80 \mathrm{ppb}$ limit has been most frequently exceeded in 2003 at both sites. However, the pollution indicator values at both stations are small and small changes from the average behavior may lead to large relative deviations. The two remaining stations Neuglobsow (3) and Vezin (2) are separated by a larger distance of ca. $530 \mathrm{~km}$ and experience obviously different conditions. At Vezin (2) the year 2003 was unusual. The value of $80 \mathrm{ppb}$ for ozone has been exceeded for a total of $106 \mathrm{~h}$ which is more than three times the average duration given in Table 2. At Neuglobsow the unusual year was 2000 with ozone being in excess of $80 \mathrm{ppb}$ for $57 \mathrm{~h}$, as opposed to years 1998 and 2001 when this happened for only 2 and $1 \mathrm{~h}$, respectively.

At K-puszta the total time when ozone exceeded the value of $80 \mathrm{ppb}$ amounted to 96 and $156 \mathrm{~h}$ in the years 1997 and 1998, respectively. In later years the values were much lower.

It is up to the experts in charge of the stations to give an explanation of observed differences which are often not noticeable in the neighboring stations. In other words the proposed indices present a simple tool for checking the validity and internal consistency of the data and can be indicative for required improvement in the validation of data and quality assurance.

\section{Conclusions}

After several attempts two indicators based on monitoring data for ozone have been defined by trial and error in order to characterize ambient air monitoring stations. They both take into account contributions from the typical diurnal behavior of ozone and from the frequency of high values measured at a particular site. The diurnal behavior is best represented by the average of daily maximum-to-minimum ratios for a season. The frequency of peak values was represented either by the average of daily maximum values or by the duration in hours when the air quality limit of $80 \mathrm{ppb}$ was exceeded. The first method is 
simpler but gives less weight to peak values than the second. The second gave a better classification of the investigated sites and provides information on the effect related peak values.

The use of the indicators is twofold: (1) they can be used to indicate what kind of photochemical pollution can be expected at a particular site and hence what effects can be expected in the neighboring regions and (2) they can be used for checking the consistency of data at a monitoring site and for a quick and simple comparison to data from neighboring sites. It has to be kept in mind that such indicators are only rough estimates which have to be used as such in order to draw attention to interesting points which should be examined in detail on the basis of all actually measured parameters at a particular site.

Acknowledgement The authors acknowledge the financial support by the Ministry of Science, Education and Sports of the Republic of Croatia (Grant no. 0098-030).

\section{References}

Butković, V., Cvitaš, T., \& Klasinc, L. (1990). Photochemical ozone in the Mediterranean. The Science of the Total Environment, 99, 145-151. doi:10.1016/ 0048-9697(90)90219-K.

Chen, T.-M., Gokhale, J., Shofer, S., \& Kuschner, W. G. (2007). Outdoor air pollution: Ozone health effects. The American Journal of the Medical Sciences, 333(4), 244-248. doi:10.1097/MAJ.0b013e31803b8e8c.

Cvitaš, T., Kezele, N., Klasinc, L., \& Lisac, I. (1995). Ozone measurements in Croatia. Pure and Applied Chemistry, 67, 1450-1453.

Fischer, P. H., Brunekreef, B., \& Lebret, E. (2004). Air pollution related deaths during the 2003 heat weave in the Netherlands. Atmospheric Environment, 38, 1083 1085. doi:10.1016/j.atmosenv.2003.11.010.

Jeftić, J., \& Cvitaš, T. (1991). Analysis of ozone monitoring data. Journal of Mathematical Chemistry, 8, 283-289. doi:10.1007/BF01166943.

Ko, F. W. S., Tam, W., Wong, T. W., Lai, C. K. W., Wong, G. W. K., Leung, T.-F., et al. (2007). Eff ects of air pollution on asthma hospitalization rates in different age groups in Hong Kong. Clinical and Experimental Allergy, 37, 1312-1319. doi:10.1111/j.1365-2222.2007. 02791.x.

Maitre, A., Bonneterre, V., Huillard, L., Sabatier, P., \& de Gaudemaris, R. (2006). Impact of urban atmospheric pollution on coronary disease. European Heart Journal, 27, 2275-2284. doi:10.1093/eurheartj/ehl162.

McNaught, A. D., \& Wilkinson, A. (1997). Compendium of chemical terminology. Oxford: Blackwell Science.

Musselman, R. C., \& Massman, W. J. (1999). Ozone flux to vegetation and its relationship to plant response and ambient air quality standards. Atmospheric Environment, 33, 65-73. doi:10.1016/S1352-2310(98)00127-7.

Ruidavets, J.-B., Cournot, M., Cassadou, S., Giroux, M., Meybeck, M., \& Ferrières, J. (2005). Ozone air pollution is associated with acute myocardial infarction. Cicrulation, 111, 563-569. doi:10.1161/01.CIR. 0000154546.32135.6E.

Sarnet, J. A., \& Holguin, F. (2007). Asthma and air quality. Current Opinion in Pulmonary Medicine, 13, 63-66.

Stedman, J. R. (2004). The predicted number of air pollution related deaths in the UK during the August 2003 heatwave. Atmospheric Environment, 38, 1087-1090.

Wayne, R. P. (2000). Chemistry of the atmospheres (3rd ed., pp. 422-439). Oxford: Oxford University Press.

West, J. J., Szopa, S., \& Hauglustaine, D. A. (2007). Human mortality effects of future concentrations of tropospheric ozone. Comptes Rendus Geoscience, 339, 775-783. doi:10.1016/j.crte.2007.08.005.

Zanobetti, A., \& Schwartz, J. (2008). Mortality displacement in the association of ozone and mortality: An analysis of 48 cities in the United States. American Journal of Respiratory and Critical Care, 177, 184-189. doi:10.1164/rccm.200706-823OC. 